\title{
Instrucción explícita e implícita en estudiantes de inglés en la formación de adjetivos
}

\author{
Explicit and implicit instruction in English learners when studying adjective formation \\ María C. García-Ramos ${ }^{a}$, Ma. De Lourdes Rico-Cruz ${ }^{b}$, Delphine Pluvinet ${ }^{c}$
}

\begin{abstract}
:
Teaching word formation is an aspect which is not often studied in language classes, but it is intended to be acquired implicitly by the students. This brings an incorrect word use and word production both in speaking and writing skills. This article has the aim of disclosing the effects of two teaching methodologies to learn suffixes in adjectives. The study was carried out with the participation of two groups of Intermediate English Language University Students. Explicit Instruction was used with one group while implicit instruction was applied with the other one through the hypertext tool, in order to use technology in the language classroom. Results of the study showed that the best effect, to learn suffixes, is found in an explicit instruction methodology despite the technological influence on the current students. Therefore, it can be sustained that, word formation teaching is an aspect which should not be pretended to be acquired totally through an implicit way.
\end{abstract}

\section{Keywords:}

Implicit instruction, explicit instruction, hypertext, word formation, adjective suffixes

\section{Resumen:}

La enseñanza de la formación de palabras es un aspecto que difícilmente se estudia como un tema en específico en la clase de lenguas; normalmente, se pretende que los estudiantes lo adquieran implícitamente. Esto resulta en una producción y los usos incorrectos de las palabras. El artículo tiene el propósito de dar a conocer los efectos de dos métodos didácticos para aprender los sufijos en adjetivos. El estudio se llevó a cabo en dos grupos de estudiantes universitarios de lengua inglesa a nivel intermedio. Los resultados mostraron que el mejor efecto para el aprendizaje de los sufijos para la formación de adjetivos se encuentra en un método de instrucción explícita a pesar de la influencia tecnológica en los estudiantes actuales. Se puede entonces corroborar que, la enseñanza de la formación de palabras es un aspecto que no se debe pretender que los estudiantes lo adquieran completamente de manera implícita.

\section{Palabras Clave:}

Instrucción implícita, instrucción explícita, hipertexto, formación de palabras, sufijos en adjetivos

\section{INTRODUCCIÓN}

En el contexto urbano, los estudiantes actuales viven cada vez más con una densa influencia tecnológica, tanto en el ámbito de su vida personal como en el educativo. Para cada situación en la que se hace uso de la tecnología se pueden identificar ventajas y desventajas. En el campo de la docencia, de igual manera, se fomenta cada día más el uso de la tecnología, sin embargo, es necesario que, como docentes, exploraremos las herramientas tecnológicas en el aula, como medio de aprendizaje, y analizar hasta qué punto pueden ser útiles para abordar temas académicos y utilizarlas como estrategias didácticas, es decir, como una verdadera ayuda pedagógica para facilitar y lograr aprendizajes significativos.

En cuanto a la enseñanza de la gramática, ahora en día, se pretende o se ha venido enseñando de manera implícita teniendo como objetivo lograr que los estudiantes se comuniquen en la lengua meta de manera "natural". Si bien es cierto que comunicarse oralmente es una habilidad esencial que un estudiante de lengua busca y necesita desarrollar, los profesores de lengua al mismo tiempo, necesitamos también considerar que esta comunicación debe ser adecuada a las normas propias de la lengua, las cuales no debemos pasar por alto.

\footnotetext{
${ }^{a}$ Correspondence Author, Universidad Autónoma de Querétaro, Facultad de Lenguas y Letras, https://orcid.org/0000-0003-4138-3389, Email: conchis_tb@hotmail.com

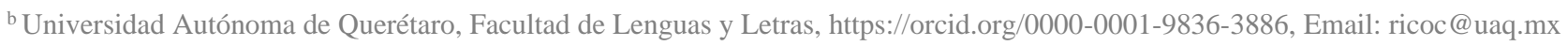

Universidad Autónoma de Querétaro, Facultad de Lenguas y Letras, https://orcid.org/0000-0001-7083-8691, Email: 
Este documento hace referencia a un problema detectado en los estudiantes de nivel intermedio de inglés en el uso de la formación de palabras. En este nivel es común observar que la producción escrita y oral se ven acompañados del uso de palabras inadecuadas. Para ayudar a los estudiantes a aumentar su conocimiento y uso en la formación de palabras, se optó por llevar a cabo un estudio de investigación para indagar cuál es la manera más adecuada de enseñar la formación de palabras y en este caso, de adjetivos.

Esta investigación se llevó a cabo con alumnos de inglés a un nivel intermedio cuyo objetivo era explorar los efectos de dos metodologías utilizadas en la clase de lengua para aprender los sufijos en los adjetivos e identificar cuál se debería adoptar para maximizar el proceso de aprendizaje en la formación de estos. En este trabajo, se aplicó la metodología implícita con el uso del hipertexto en actividades pedagógicas con un grupo de estudiantes y una metodología explicita en otro grupo de estudiantes, donde el profesor de manera directa enfatiza el uso y significado de ciertos sufijos para la formación de adjetivos. Para Kommers \& Lanzing, (1997) con el hipertexto hay una actividad en donde se integran una serie de conocimientos, los cuales comprenden facetas cognitivas y metacognitivas que pasan a formar parte del proceso de aprendizaje. En educación, se ha usado el hipertexto como un tipo de lectura no secuencial; como una estrategia didáctica que se sirve de un sistema implícito, en el que el alumno no es consciente de que está adquiriendo normas de construcción gramatical, es decir, las aprende de forma intuitiva; la información se deriva de un modo inconsciente, no reflexivo (Jorge Martí, 2015, p:8).

Por otro lado, trabajar la gramática en el proceso de aprendizaje de lenguas a través del eje instruccional explícito, consiste en la exposición de las reglas a los alumnos por parte del profesor, de esta manera, se pretende así que la gramática se adquiera de manera consciente (Rod Ellis (1994).

\section{REVISIÓN DE LA LITERATURA}

\subsection{El hipertexto como recurso didáctico}

El hipertexto debe ser pensado principalmente como un modelo mediante el cual es posible pensar y representar un sistema de relaciones uno a uno, uno-muchos y muchos-muchos (Gamba Bar, 2004:3).

El hipertexto predispone a una lectura y escritura no lineal. La idea de secuencialidad se reemplaza por la idea de interrelación, por lo que el lector construye con su lectura (Ken, Beatty, 2010:42). Pajares Tosca (2004) propone que el hipertexto es una estructura de base informática para organizar información que hace posible la concepción electrónica de unidades textuales (de diferente tamaño y categoría) a través de enlaces (links) dentro de un mismo documento o con documentos externos; los cuales requieren de la manipulación activa del lector para poder ser leído / utilizado.

Roland Barthes (1995: 11) define que "el hipertexto electrónico es un texto compuesto de bloques de palabras o imágenes electrónicamente unidos en múltiples trayectos, cadenas o recorridos en una textualidad abierta, eternamente inacabada y descrita con términos como nexo, nodo, red, trama y trayecto". Las actividades diseñadas para esta investigación se basan en el concepto de hipertexto antes mencionado, sin embargo, este trayecto ha sido semi -controlado para no dar oportunidad a que los estudiantes se enfrentaran a desviaciones debido a la exposición de excesivo número de enlaces electrónicos (links) para acudir a la información sabiendo que éste tiene diversas vías de acceso, sin que ninguna de ellas pueda calificarse como la principal. Si comparamos un salón de clase con los enlaces electrónicos, podemos encontrar semejanzas dado que en una clase frecuentemente se presentan referencias a situaciones de la vida real y experiencias que los estudiantes han pasado, o se les provee o solicita ejemplos acerca del tema, es decir; estamos de una o de otra manera frecuentemente saltando del tema a imágenes o situaciones mentales, aunque no sean electrónicas. El uso del hipertexto como estrategia didáctica, ha sido utilizado y analizado en la enseñanza de lenguas. Como ejemplo podemos citar a Kang (2010) quien llevó a cabo un estudio en el que se investigaron las estrategias de lectura que los estudiantes angloparlantes de chino, nivel avanzado, utilizaban al leer diferentes géneros, tales como ensayos, periódicos, poemas o prosa, considerando dos formatos, uno basado en papel y el otro en computadora utilizando el hipertexto para ir a diccionarios y glosarios en línea. El estudio pretendía identificar cuáles eran los patrones que los estudiantes de chino utilizaban al leer textos en lengua extranjera según el tipo de texto. En tal estudio se encontró que se distraían con los nodos en los periódicos y otros textos en prosa, lo que llevó a confundirlos porque empezaban a leer la primera parte de un párrafo y movían sus ojos a la información en los nodos, lo cual causaba distracción del objetivo principal de la actividad asignada. Se puede aquí ver que el hipertexto, como herramienta didáctica, se debe utilizar con cautela para verificar que efectivamente el estudiante siga un proceso de actividad académica y no se desvíe del propósito. De igual forma, María Cristina (2010) realiza un estudio sobre estrategias de comprensión con el uso del hipertexto informativo en lectores competentes de internet mientras navegan con el propósito de resolver una tarea asignada basada en la comprensión. Los resultados mostraron que un individuo competente es capaz de combinar eficiente y efectivamente el conocimiento previo que posee del tema que lee y de la estructura del texto expositivo con el buen manejo de la estructura del hipertexto.

Si bien, se ha utilizado el hipertexto como estrategia didáctica principalmente para la comprensión de lectura, no ha sido para abordar temas gramaticales, y es aquí en donde se ha aprovechado la oportunidad para investigar sobre el efecto de esta herramienta en la enseñanza implícita de la gramática. Se tomó esta decisión debido a que el hipertexto puede incluir actividades de información visual, lectora y auditiva considerando los diferentes estilos de aprendizaje que se pueden encontrar en el salón de clase. De esta forma, "en un hipertexto, no solo tenemos acceso no lineal a pedazos de texto, sino una 
diversidad riquísima de estímulos materializados en imágenes, ya sea en fijas o en movimiento- en sonidos, en animaciones o en cualquier otra forma de manifestación informática" Calderoni, José \& Pacheco, Valeria (1998:161).

\subsection{La instrucción explícita}

Trabajar la gramática en el proceso de enseñanza- aprendizaje de lenguas se puede abordar de dos maneras distintas. Una de ellas supone un trabajo mayor meta cognitivo y reflexivo de parte del estudiante de tal forma que se apodere de las estructuras lingüísticas sin verse inmerso en una explicación tan directa del tema por parte del docente, sino que más bien de una manera inconsciente la aprenda, mientras que en la segunda el docente presenta el tema de una forma explícita en el aula. "Ésta se basa en la exposición de las reglas a los alumnos por parte del profesor, se busca así que la gramática se adquiera conscientemente" (Ellis, 1994:643).

Algunas de las características del aprendizaje explícito de la gramática, según Gómez del Estal (2004) son que los alumnos analizan las reglas gramaticales, que el alumno puede acceder y hablar sobre las reglas sin esfuerzo. A su vez, Latinjak, Alexander (2014, p:4) establece que el aprendizaje explícito es todo aquel aprendizaje en el cual el aprendiz tiene intención de aprender y es consciente de qué aprende.

Todo lo expuesto acerca de la instrucción explícita es lo que hace la diferencia, en esta investigación, con el uso del hipertexto. Esto no significa que el hipertexto sea exclusivamente para enseñar y aprender gramática de forma implícita, sino que, se usó la herramienta tecnológica en esta ocasión como medio para enseñar la lengua de forma implícita, y para dar cuenta del efecto de ambas metodologías exclusivamente en la enseñanza de los sufijos mediante este sistema en el que el alumno no es consciente de que está adquiriendo normas de construcción gramatical, sino que las aprende de manera intuitiva. Se define enseguida a qué se refiere con enseñanza implícita.

\subsection{La instrucción implícita}

Reben, Arthur (1989, p:2) acuña el término de aprendizaje implícito como "el proceso por el cual el conocimiento se adquiere de manera independiente de la conciencia. El aprendizaje implícito produce una base de conocimiento tácito en el que se abstrae la estructura, tal conocimiento es adquirido de manera independiente a los esfuerzos consientes para aprender y puede utilizarse implícitamente para solucionar problemas". La conciencia es la médula en este trabajo de investigación, verificar hasta dónde es posible lograr que el estudiante aprenda de manera consiente e inconsciente con las metodologías ya descritas.

Muchos docentes queremos ver el logro del aprendizaje en los estudiantes, sin embargo, se requiere poner cuidado a cuál metodología se debe seguir de acuerdo al tema de enseñanza. Aunque la tecnología (el hipertexto) sea una estrategia moderna para enseñar lenguas, es necesario considerar si aprender sufijos en adjetivos también puede ser abordado de manera inconsciente o si requiere de una explicación más profunda y una enseñanza consciente para el estudiante.
Lo expuesto acerca de los dos enfoques de enseñanza hace conexión a las palabras de Ellis, Rod (2003, p:533) en cuanto a tres etapas o formas de estrategias de aprendizaje. 1) la etapa cognitiva, en donde el aprendiz se involucra en actividades de manera consciente; 2) la etapa de asociación, en donde el aprendiz fortalece las conexiones entre los componentes de la habilidad y construye una producción más eficiente; 3) la etapa automática, en donde la ejecución se convierte más o menos autónoma y subconsciente. La primera etapa hace referencia a la enseñanza explícita mientras que la tercera puede relacionarse con la enseñanza implícita que se abordan en este estudio.

\section{METODOLOGÍA}

El tipo de investigación que aquí se presenta es de carácter exploratorio y cuantitativo. Busca identificar la efectividad de dos metodologías para la enseñanza de los sufijos en adjetivos de la lengua inglesa y se realiza, de igual forma, una cuantificación de los aciertos obtenidos en un pre y un post test después de la intervención didáctica en cada tipo de ejercicios. Para la intervención didáctica se seleccionaron dos grupos de estudiantes quienes cursan un nivel intermedio de inglés en una universidad mexicana. Con uno de los grupos se trabajó con la mediación del uso del hipertexto mientras que con el otro se utilizaron explicaciones de la gramática por parte de un profesor y actividades de gramática.

Ahora bien, el primer grupo se conformó de 10 participantes cuyas edades oscilan entre 18 - 20 años. Cuentan con un manejo de la computadora y el internet a un nivel aceptable para trabajar con el hipertexto. Algunos no llevaban computadora al salón de clase, pero utilizaron sus teléfonos tanto para bajar con los archivos enviados a sus correos como para escribir en el procesador de palabras.

Las actividades se llevaron a cabo de la siguiente manera; primeramente, se realizó un ejercicio de producción oral para identificar cuál podría ser un tema de dificultad común para investigar y en el que se pudiera aplicar una estrategia didáctica para ayudar a los estudiantes de este nivel. Una vez identificados los problemas, se eligió uno y se decidió trabajar con la formación de palabras, exclusivamente con algunos sufijos para los adjetivos en la lengua inglesa. Estos sufijos son: -ful, -less, $e d$, -ing, -ive, \& -able. La intervención tuvo diferentes momentos los cuales se describen en la siguiente sección.

\subsection{Grupo con Enseñanza Implícita a través del Hipertexto}

Con el grupo A, se llevó a cabo la aplicación de un pre test para compararlo con un post test e identificar el efecto de la estrategia didáctica. El pre test consistió en dos ejercicios, el primero enfocado exclusivamente para completar oraciones con la formación de la palabra apropiada de acuerdo a la palabra dada al final de la línea, con el objetivo de verificar si sabían utilizar los sufijos pertinentes. En el ejercicio dos se les dio una lista de palabras para marcar sólo las que tuvieran la forma de un adjetivo con el propósito de verificar si se tiene la capacidad de discernir entre un sustantivo, un verbo o un adjetivo. 
Las actividades aplicadas después del pre test están basadas en una secuencia didáctica contextualizada de tal forma que la lengua se enseñe de manera implícita utilizando el hipertexto. El contexto gira alrededor del tópico "shopping" (ir de compras). Esta intervención tiene una duración de 4 horas (4 sesiones cada una).

En la sesión 1 consistió en enviar a los estudiantes una liga a sus correos para entrar al sitio web y ver un video relacionado con lugares para ir de compras (stores). Los estudiantes describen las tiendas, personas y artículos que observan en el video utilizando adjetivos con terminaciones, -ful, -less, -ed, -ing, -ive, \& -able de una lista dada en un sitio web al que también deben dar click. Una vez completada la descripción es enviada a sus compañeros de clase, utilizando el correo electrónico.

En la sesión 2, se distribuyó un texto escrito relacionado con el tema en donde encuentran palabras subrayadas (adjetivos), con las terminaciones antes descritas, y a las cuales deben dar click para leer las definiciones de cada una con el propósito de aprender sufijos de manera inconsciente.

En la sesión 3 se completaron tres actividades diferentes. En la primera actividad, los estudiantes trabajaron con un texto incompleto en el que debían generar la palabra que faltaba en la línea (adjetivo) de acuerdo a la palabra dada en mayúscula al final de ésta. Se les proporcionó ayuda para imaginar cuál es la palabra faltante. Los participantes debían dar click en la palabra en mayúscula para ir a una imagen que servía de clave para pensar en la palabra que completaba la frase. En la segunda actividad, los estudiantes, de igual forma completaron un texto semejante al anterior, la diferencia yace en el tipo de clave para completar con el adjetivo faltante. Al dar click en la palabra subrayada observaban la traducción del adjetivo en su lengua madre, español, con el propósito de reflexionar en cuál sería la terminación que se necesita en la lengua inglesa. La tercera actividad consistió en una dinámica semejante a las anteriores, la diferencia estuvo en la clave para pensar en el adjetivo. Al dar click en la palabra subrayada los participantes fueron llevados a ver y escuchar un video de una conversación no mayor a 3-4 minutos. La actividad consistió en dar pausa al video justo en el momento en que escucharan la palabra (adjetivo) que necesitan para completar la frase en el texto. Se les permitió escuchar las veces que fueran necesarias.

En la última sesión, los estudiantes leyeron y escucharon un texto sobre lugares para ir de compras en Londres, con el propósito de desarrollar un escrito de 180-200 palabras sobre las ventajas y desventajas de comprar en Londres y en su país, citando los mejores lugares de acuerdo a la lectura y utilizando los adjetivos previamente estudiados en las sesiones anteriores cuyas terminaciones fueran -ful, -less, -ed, -ing, -ive, \& -able.

Posteriormente a la intervención pedagógica, se aplicó un posttest (mismo que el pre-test). Con el objetivo de comparar la diferencia en los resultados iniciales y los finales después de la intervención. Para concluir la investigación, se aplicó de forma oral una entrevista a los participantes con el propósito de identificar si recordaban algunos adjetivos de los estudiados en las sesiones, así como también para conocer acerca de las formas en las que habían estudiado los sufijos anteriormente y saber sobre sus opiniones de esta forma de enseñanza. Toda esta información fue grabada para su análisis.

\subsection{Grupo con Enseñanza Explícita}

El segundo grupo estuvo formado por 6 participantes, estudiantes de inglés intermedio de un centro de lenguas en una universidad mexicana, cuyas edades oscilan entre los 20-50 años de edad.

Se aplicó a los participantes un pre-test (mismo examen que con la enseñanza implícita). Dicho examen consiste en dos ejercicios de 30 reactivos cada uno. En el primer ejercicio los estudiantes deben generar la palabra (adjetivo) y en el segundo solamente se debe identificar y marcar cuáles palabras son adjetivos.

Posteriormente, la intervención con la enseñanza de los sufijos en adjetivos se basó en la misma lista de sufijos que con la estrategia con el hipertexto, ful, -less, -ed, -ing, -ive, \& -able. La secuencia didáctica para esta clase se desarrolla bajo el tema "The Digestive System" (El sistema digestivo) y cuenta con 4 sesiones de una hora cada una y fueron grabadas con video y audio.

En la sesión 1, el investigador elicita qué es lo que han comido esa mañana y de dónde proviene la energía que tiene su cuerpo (para introducir al tema del aparato digestivo). Se da la oportunidad a cada estudiante para mencionar la lista de productos que han ingerido y discriminar cuáles les dan energía. En esa sesión, los participantes etiquetan una imagen de un sistema digestivo con vocabulario otorgado. Luego, entran a un sitio web proporcionado por el investigador y buscan en esa lista de palabras (adjetivos) las que puedan ser útiles para describir el sistema digestivo. La actividad es controlada dado que solo debe utilizar palabras (adjetivos) que terminen en ful, -less, -ed, -ing, -ive, \& -able. Finalmente, los participantes escriben los adjetivos en el pizarrón y se les pide que subrayen la raíz de esa palabra y luego el sufijo que le corresponde. Por último, el investigador ayuda a los estudiantes a identificar la raíz y sufijo para los casos difíciles y comienza a explicar qué significa el sufijo de cada adjetivo escrito en el pizarrón.

Por ejemplo: - full= means "a lot ", - less= means "without", - ed= describes a feeling or an emotion, - ing= describes a characteristic of a person, a thing or a situation, - ive= means that does or tends to do a specified, - able= means having sufficient power, skill or resources.

En la sesión 2, a través de un video en silencio y explicación del investigador, se trabaja solo con los sufijos "-ful" y "-less". Se pausa el video, se explican las palabras con su respectivo significado y se dan ejemplos con los que trabaja el estudiante. Así mismo, en la sesión 3, se realiza un repaso de los dos sufijos anteriores y se recuerdan los adjetivos estudiados. Se trabaja con la explicación y ejemplos de adjetivos únicamente con terminaciones "-ed" e "ing" utilizando video y la explicación del investigador. Los estudiantes practican completando una conversación. 
En la sesión 4, se hace un repaso de los sufijos anteriores, su significado y recordando ejemplos de cada uno. Se trabaja, de igual forma a través de un video y explicación del docente, con los sufijos "ive y able". Se dan ejemplos y los estudiantes practican en fotocopias. Después de la serie de actividades realizadas se procede a la realización de un escrito por parte de los participantes en el que se les pide que describan el sistema digestivo utilizando adjetivos utilizados en clase y cuyos sufijos sean ful, -less, -ed, -ing, -ive, \& -able. El propósito de la actividad es analizar y cuantificar el uso de los adjetivos con los sufijos en la investigación.

Al igual que en la enseñanza implícita con la mediación del hipertexto, se aplica un post-test (mismo examen que el inicial) con la finalidad de observar la diferencia en los resultados antes y después de la intervención pedagógica. Finalmente se aplicó una entrevista de forma oral a los participantes con el propósito de identificar si recordaban algunos adjetivos de los estudiados en las sesiones, así como también para conocer acerca de las formas en las que habían estudiado los sufijos anteriormente y saber sobre sus opiniones de esta forma de enseñanza. Toda esta información fue grabada para su análisis.

\section{RESULTADOS}

En este artículo sólo se consideran los resultados del examen previo a la intervención y los del examen final. A continuación, se muestra numérica y gráficamente el total de aciertos en cada actividad del examen. El primer par de tablas muestran los aciertos de la actividad 1 en ambos grupos, con los dos tipos de intervención.

\begin{tabular}{|l|c|c|c|}
\hline \multicolumn{2}{|c|}{ ACTIVIDAD 1 } & CON HYPERTEXTO & \\
\hline & $\begin{array}{c}\text { Total aciertos } \\
\text { PRE TEST }\end{array}$ & $\begin{array}{c}\text { Total aciertos } \\
\text { Post Test }\end{array}$ & $\begin{array}{c}\text { Total aciertos } \\
\text { mejorados }\end{array}$ \\
\hline P1 & 18 & 19 & 1 \\
\hline P2 & 1 & 2 & 1 \\
\hline P3 & 23 & 24 & 1 \\
\hline P4 & 13 & 17 & 4 \\
\hline P5 & 17 & 21 & 4 \\
\hline P6 & 16 & 24 & $\mathbf{8}$ \\
\hline P7 & 15 & 14 & 0 \\
\hline P8 & 2 & 1 & 0 \\
\hline P9 & 16 & 21 & $\mathbf{5}$ \\
\hline P10 & 24 & 24 & $\mathbf{0}$ \\
\hline
\end{tabular}

\begin{tabular}{|l|c|c|c|}
\hline \multicolumn{3}{|l|}{ ACTIVIDAD1 ENSENANZA EXPLICITA } & \\
\hline & & \\
\hline & $\begin{array}{c}\text { Total aciertos } \\
\text { PRE TEST }\end{array}$ & $\begin{array}{c}\text { Total aciertos } \\
\text { Post Test }\end{array}$ & $\begin{array}{c}\text { Total aciertos } \\
\text { mejorados }\end{array}$ \\
\hline P1 & 6 & 11 & 5 \\
\hline P2 & 14 & 21 & 7 \\
\hline P3 & 13 & 20 & 7 \\
\hline P4 & 10 & 15 & 5 \\
\hline P5 & 11 & 23 & 12 \\
\hline P6 & 10 & 11 & 1 \\
\hline
\end{tabular}

Tabla 1 Total de aciertos en el Pre test y en el Post Test

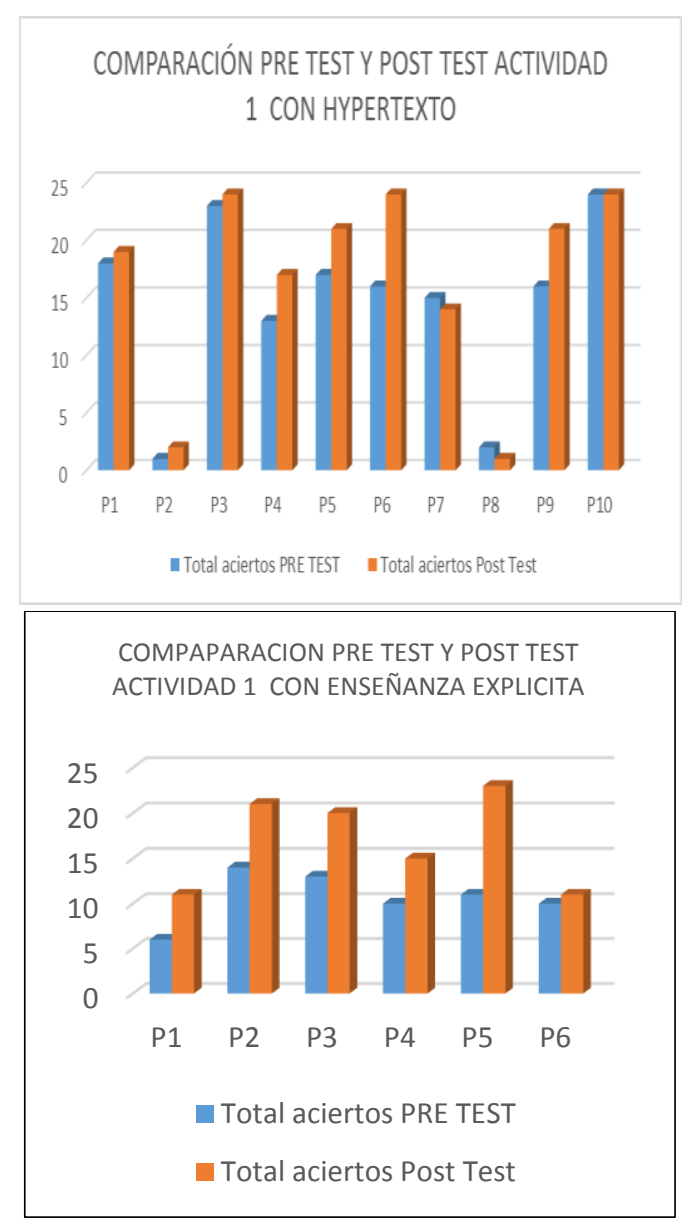

Gráfica 1 Total de aciertos en el Pre test y en el Post Test

Para la lectura de éstos resultados se utilizó la estadística descriptiva y a través de una prueba $\mathrm{T}$ se muestra que sí es significativa la diferencia entre los resultados de la actividad 1 en el pre test y el post test con ambas metodologías, sin embargo, se aprecia que hubo mejores resultados con la intervención de la enseñanza explícita, el resultado p0.00853215 es mucho menor que el alfa de 0.05 .

\section{Con Enseñanza Implícita}

\begin{tabular}{|c|c|c|}
\hline & \multicolumn{2}{|c|}{ ENSEÑANZA CON HIPERTE } \\
\hline \multicolumn{3}{|c|}{ Prueba t para medias de dos muestras } \\
\hline \multicolumn{2}{|c|}{ Total aciertos PRE } & aciertos Post \\
\hline Media & 14.5 & 16.7 \\
\hline Varianza & 58.5 & 74.6777778 \\
\hline Observacion & 10 & 10 \\
\hline Coeficiente । & 0.94223554 & \\
\hline Diferencia hi & 0 & \\
\hline Grados de lik & 9 & \\
\hline Estadístico t & -2.36926192 & \\
\hline $\mathrm{P}(\mathrm{T}<=\mathrm{t})$ una $\mathrm{c}$ & 0.02097984 & \\
\hline Valor crítico & 1.83311293 & \\
\hline $\mathrm{P}(\mathrm{T}<=\mathrm{t}) \operatorname{dos} \mathrm{C}$ & 0.04195968 & \\
\hline Valor crítico & 2.26215716 & \\
\hline
\end{tabular}


Con Enseñanza Explícita

\begin{tabular}{|c|c|c|}
\hline & \multicolumn{2}{|c|}{ ENSEÑANZA EXPLICITA } \\
\hline \multicolumn{3}{|c|}{ Prueba t para medias de dos muestras } \\
\hline \multicolumn{3}{|c|}{ Total aciertos PRE aciertos Post } \\
\hline Media & 10.6666667 & 16.8333333 \\
\hline Varianza & 7.86666667 & 27.3666667 \\
\hline Observacion & 6 & 6 \\
\hline Coeficiente & 0.75878526 & \\
\hline Diferencia hi & 0 & \\
\hline Grados de lik & 5 & \\
\hline Estadístico t & -4.19480644 & \\
\hline$P(T<=t)$ una $c$ & 0.00426608 & \\
\hline Valor crítico & 2.01504837 & \\
\hline $\mathrm{P}(\mathrm{T}<=\mathrm{t})$ dos $\mathrm{C}$ & 0.00853215 & \\
\hline Valor crítico & 2.57058184 & \\
\hline
\end{tabular}

Tabla 2. Prueba $T$ reflejando la significatividad de la diferencia entre los aciertos del pre test y el post test en cada grupo.

En cuanto a la actividad 2 de ambos exámenes, también se utiizó la estadística descriptiva con la prueba T para leer los resultados y ver si hay significancia en la diferencia de éstos en el uso de los adjetivos con sufijos después de la intervención pedagógica con las dos formas de enseñanza, como se muestra en la tabla 2.

\begin{tabular}{|c|c|c|c|}
\hline & \multicolumn{3}{|c|}{ ACTIVIDAD 2 ENSEÑANZA EXPLICITA } \\
\hline \multicolumn{4}{|c|}{ Underline the words which are adjectives } \\
\hline \multicolumn{4}{|c|}{30 palabras en la lista. 16 adjetivos y 14 sustantivos } \\
\hline \multicolumn{4}{|c|}{ GRUPO RECIENTE } \\
\hline PRE TEST & $\begin{array}{c}\text { Total aciertos } \\
\text { PRE TEST }\end{array}$ & $\begin{array}{c}\text { Total aciertos } \\
\text { Post Test }\end{array}$ & $\begin{array}{c}\text { Total aciertos } \\
\text { mejorados }\end{array}$ \\
\hline P1 & 12 & 6 & 4 \\
\hline P2 & 12 & 16 & 4 \\
\hline P3 & 9 & 14 & 5 \\
\hline P4 & 12 & 15 & 3 \\
\hline P5 & 10 & 15 & 5 \\
\hline P6 & 7 & 13 & 6 \\
\hline & & & \\
\hline & & & \\
\hline & & & \\
\hline & & & \\
\hline
\end{tabular}

\section{ACTIVIDAD 2 CON HIPERTEXTO}

Underline the words which are adjectives

30 palabras en la lista. 16 adjetivos y 14 sustantivos

\begin{tabular}{|l|c|c|c|}
\hline PRE TEST & $\begin{array}{c}\text { Total aciertos } \\
\text { PRE TEST }\end{array}$ & $\begin{array}{c}\text { Total aciertos } \\
\text { Post Test }\end{array}$ & $\begin{array}{c}\text { Total aciertos } \\
\text { mejorados }\end{array}$ \\
\hline P1 & 8 & 13 & $\mathbf{5}$ \\
\hline P2 & 12 & 15 & $\mathbf{3}$ \\
\hline P3 & 7 & 14 & $\mathbf{7}$ \\
\hline P4 & 10 & 12 & $\mathbf{2}$ \\
\hline P5 & 14 & 15 & $\mathbf{1}$ \\
\hline P6 & 12 & 14 & $\mathbf{2}$ \\
\hline P7 & 11 & 15 & $\mathbf{4}$ \\
\hline P8 & 11 & 6 & $\mathbf{0}$ \\
\hline P9 & 10 & 13 & $\mathbf{3}$ \\
\hline P10 & 15 & 15 & $\mathbf{0}$ \\
\hline
\end{tabular}

Tabla 3 Total de aciertos en el Pre test y en el Post Test
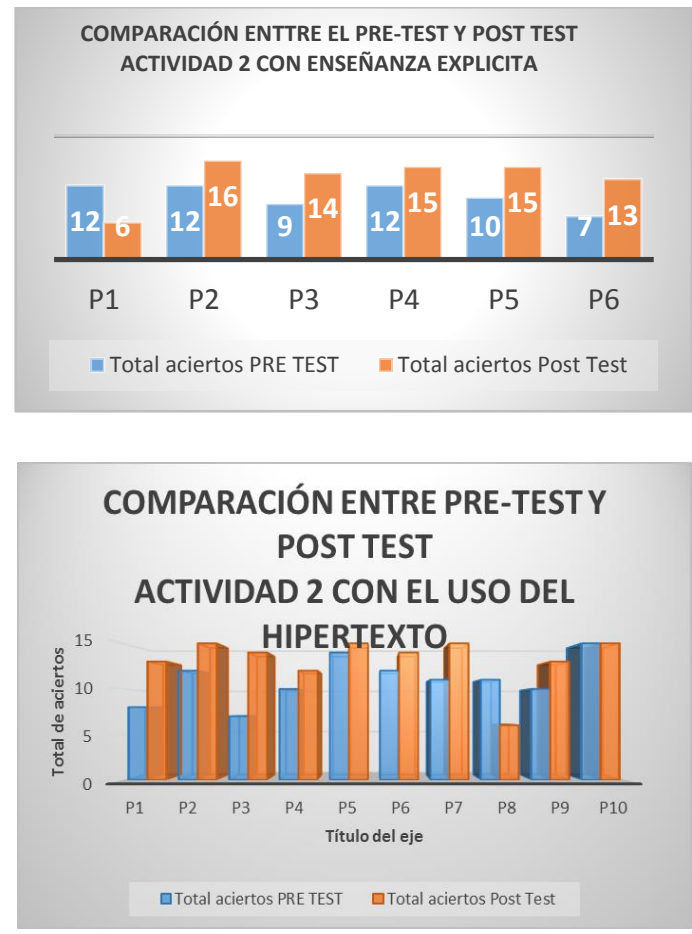

Gráfica 2 Total de aciertos en el Pre test y en el Post Test

A partir de un análisis en los dos exámenes (pre-test y post test) y utilizando la estadística descriptiva para saber qué está pasando, se utilizó una prueba $\mathrm{T}$ en la que para la actividad 1 del examen, donde tienen que generar la palabra para completar la frase, se muestra que la estrategia tanto de hipertexto como en la enseñanza explícita sí han sido significativas ya que se puede ver una diferencia en los puntajes de la tabla 1 y se confirman en la tabla 2 y la gráfica con los datos estadísticos. Sin embargo, éstas mismas muestran que hubo una mayor diferencia en los puntajes con la aplicación de la enseñanza explícita. Esto refleja que, el tema necesita de este tipo de estrategia para ser enseñado dado que la formación de palabras no es un tema sencillo y que el estudiante necesita conocer cuál es el significado de cada sufijo para que esté seguro del significado que le hace falta a la palabra que debe completar la frase.

\section{Con Enseñanza Implícita}

Prueba t para medias de dos muestras emparejadas

\begin{tabular}{|l|r|r|}
\hline \multicolumn{3}{|c|}{ Total aciertos PRE } \\
\hline Mediertos Post Test \\
\hline Varianza & 10.3333333 & 13.1666667 \\
\hline Observacion & 4.26666667 & 13.3666667 \\
\hline Coeficiente 1 & -0.14124491 & \\
\hline Diferencia hi & 0 & 6 \\
\hline Grados de lit & 5 & \\
\hline Estadístico t & -1.5610132 & \\
\hline$P(T<=t)$ una c & 0.08963658 & \\
\hline Valor crítico & 2.01504837 & \\
\hline$P(T<=t)$ dos c & 0.17927315 & \\
\hline Valor crítico & 2.57058184 & \\
\hline
\end{tabular}




\section{Con Enseñanza Explícita}

\begin{tabular}{|l|r|r|}
\hline \multicolumn{2}{|l|}{ Prueba t para medias de dos muestras emparejadas } \\
\hline \multicolumn{3}{|c|}{ Total aciertos PRE } \\
\hline Mediartos Post Test \\
\hline Varianza & 11 & 13.2 \\
\hline Observacion & 6 & 7.51111111 \\
\hline Coeficiente r & 0.23171695 & 10 \\
\hline Diferencia hi & 0 & \\
\hline Grados de lit & 9 & \\
\hline Estadístico t & -2.15727749 \\
\hline$P(T<=t)$ una c & 0.02966491 \\
\hline Valor crítico & 1.83311293 \\
\hline$P(T<=t)$ dos c & 0.05932981 \\
\hline Valor crítico & 2.26215716 \\
\hline
\end{tabular}

Tabla 4. Prueba $\mathrm{T}$ reflejando la significatividad de la diferencia entre los aciertos del pre test y el post test en cada grupo.

En cuanto a la actividad 2 de los dos exámenes, se observa en la tabla 2 y se confirma en los datos estadísticos en la tabla 4 que, la estrategia de enseñanza explícita tuvo un mayor efecto que en el grupo con enseñanza a través del hipertexto. Así mismo se refleja que la estrategia de enseñanza explícita ha sido útil, sobre todo, para ayudar al estudiante a identificar, si la palabra dada es un adjetivo o no.

\section{CONCLUSIÓN}

Estos resultados tienen implicación pedagógica porque los profesores de lengua al conocer que se puede hacer uso del hipertexto o de una explicación explícita para enseñar la formación de palabras tendrá otras opciones más para experimentar formas de enseñanza distintas y atender a los estudiantes según su estilo de aprendizaje (de forma explícita o implícita).

Con el análisis se prueba que el hipertexto sirve para mejorar la competencia de la formación de palabras. Sin embargo, tiene mayor efectividad la enseñanza explícita. Se puede entonces corroborar que, la enseñanza de la formación de palabras es un aspecto que no se debe pretender que los estudiantes lo adquieran implícitamente.

\section{REFERENCIAS}

Ellis, Rod. The Study of Second Language Acquisition. Tenth. Oxford: Oxford University Press, 2003.

https://books.google.com.mx/books?hl=es\&lr=\&id=3KglibyrZ5sC\&oi=fn $\mathrm{d} \& \mathrm{pg}=\mathrm{PR} 5 \& \mathrm{dq}=\mathrm{The}+$ study + of + Second + Language + Acquisition+book + pd f\&ots $=w E Y q e m 1 F x \_\& s i g=B u-$

5lc8LkTCsYBSLcoZc7NpGvTs\#v=onepage \&q\&f=false.

Gamba, Antonio (2004). "Hipertexto y pensamiento: una búsqueda de nuevas herramientas deinterlocución por Antonio Gamba Ba", s/f. http://www.ub.edu/prometheus21/articulos/obsciberprome/Gamba.pdf.

José, Calderoni, y Pacheco, Valeria. "El hipertexto como nuevo recurso didáctico", Revista Latinoamericana de Estudios Educativos, XXVIII, núm. 3 y 4 (1998): 157-81.

http://www.redalyc.org/pdf/270/27028407.pdf.
Kang, H. (2010). A Case study: Comparing reading strategies of advanced CFL learners on different genres and media of text, 9. Recuperado de https://kb.osu.edu/handle/1811/31925

Ken Beatty. Teaching and Researching Computer-Assited Language Learning. Second. Great Britain: Pearson, 2010.

López Andrada, Concepción. (2018). La lectura del clic. Una introducción al uso didáctico del hipertexto. Centro de Estudios Latinoamericanos de Educación Inclusiva | CELEI | Chile: CELEI. Recuperado de https://www.celei.cl/wpcontent/uploads/2018/03/Documento-4_Observatorio-Educaci\%C3\%B3nLectora_CELEI_-Autor_Lopez-Andrada.pdf

María Cristina, A. (2010). Estrategias de Comprensión con Hipertetxo informativo, 31(2), 18-32. Recuperado de http://www.lecturayvida.fahce.unlp.edu.ar/numeros/a31n2/31_02_Arancib ia.pdf

Martí Contreras, Jorge. “GRAMÁTICA IMPLÍCITA O GRAMÁTICA EXPLÍCITA EN ENSEÑANZA DE SEGUNDAS LENGUAS?: ESTUDIO DE CAMPO”. Normas 0, núm. 5 (el 4 de agosto de 2015). https://doi.org/10.7203/Normas.5.6818.

Martínez, Elizabeth., \& Zea, Enilda. (2004, diciembre). "ESTRATEGIAS DE ENSEÑANZA BASADAS EN UN ENFOQUE CONSTRUCTIVISTA", 2(24), 79. Recuperado a partir de http://servicio.bc.uc.edu.ve/educacion/revista/a4n24/4-24-4.pdf

Reber, Arthur. "Implicit Learning and Tacit Knowledge", ournal of Experimental Psychology Genera, 118 (el 27 de marzo de 2014): 18. https://doi.org/10.1037/0096-3445.118.3.219.

T. Latinjack Alexander. “Aprendizaje Implícito y Explícito: Entre el Hacer y el Comprender”, 2014.

http://www3.udg.edu/publicacions/vell/electroniques/VI_Jornades_aprend izaje_accion_tactica/docs/05_cap3.pdf, file:///C:/Users/DELL/Download s/Aprendizajeimplcitoyexplcito_Entreelhacerielcomprender.pdf.

Vannevar, Bush. "As we May Think”, The Athlantic Monthly, 176, núm. 1 (1945): 1-14.

http://iibi.unam.mx/voutssasmt/documentos/Vannevar_Bush_Como\%20p odriamos\%20_Pensar_JV.pdf. 